\title{
Control strategies for the final focus of future linear particle collider
}

\author{
C. Collette*, S. Janssens, D. Tshilumba \\ Active Structures Laboratory, University of Brussels, 50, av. F.D. Roosevelt, 1050 Brussels, Belgium
}

\section{A R T I C L E I N F O}

\section{Article history:}

Received 7 February 2012

Received in revised form

24 April 2012

Accepted 29 April 2012

Available online 11 May 2012

Keywords:

Final focus

Active stabilization

Nanopositioning

Seismic vibration isolation

Active damping

Cabled structures

Hybrid systems

\begin{abstract}
A B S T R A C T
This paper presents a simple model of the final focus of a linear particle collider. Adopting an integrated approach, several control strategies are tested to stabilize the mechanical parts, and control the beam. One of the key features of the model is that it has been updated using vibration spectra measured in the CMS experimental area of the LHC. Using this model, it has been possible to estimate objectively the performances of a final focus system, compare and propose new solutions to improve the mechanical design.
\end{abstract}

(c) 2012 Elsevier B.V. All rights reserved.

\section{Introduction}

During the last 50 years, the energy and size of the particle accelerators have been multiplied by five orders of magnitude. In the future, it is foreseen to continue to explore new physics with linear particle colliders. Two projects are currently under study: the International Linear Collider (ILC) [1] and the Compact Linear Collider (CLIC) [2]. In CLIC, electrons and positrons will be accelerated in two linear accelerators to collide at the interaction point with an energy of $0.5-3 \mathrm{TeV}$ [3]. To acquire such a high energy, the total length of the machine will be $48 \mathrm{~km}$, and constituted of a very large number (more than 20000 ) of identical modules, the function of which is to accelerate and focus the beam of particles, towards the final section where the collision takes place. Hand in hand with the energy, the so-called luminosity of particle colliders (proportional to the number of collisions per second and unit area) has also followed the same historical trend, requiring to produce increasingly small, dense and stable beams [4]. In linear accelerators, the beam cross section is extremely flat, with a vertical size typically 100 times smaller than the horizontal size. Considering only the vertical direction (because it is the most critical), let us first define $\Delta y$ as the average vertical distance between the two colliding beams at

\footnotetext{
* Corresponding author.

E-mail addresses: christophe.collette@cern.ch, christophe.collette@ulb.ac.be (C. Collette).
}

the Interaction Point (IP), such as

$\Delta y=y^{+}-y^{-}$

where $y^{+}$and $y^{-}$are the positions of the two beams at the IP $[5,6]$. It can be shown that the dependency of the luminosity, $L$, with the offset $\Delta y$ is approximately given by [7]

$L \approx L_{0} e^{-\Delta y^{2} / 16 \sigma_{y}^{2}}$

where $\sigma_{y}$ is the vertical beam size at the IP and $L_{0}$ is the nominal luminosity (i.e. the luminosity in a perfect machine). For both ILC and CLIC, the nominal luminosity is $L_{0} \approx 2 \times 10^{34} \mathrm{~cm}^{-2} \mathrm{~s}^{-1}$. Eq. (2) shows that to mitigate the luminosity losses, the smaller the size of the beam, the more stable the final focus of the machine, just before the IP. For ILC, $\sigma_{y}=5.7 \mathrm{~nm}$ (and $640 \mathrm{~nm}$ in the horizontal direction). However, the permissible beam jitter is still about $50 \mathrm{~nm}[8,9]$, because it considers the possibility to recover the luminosity with an intra-pulse feedback [10]. As a comparison, for CLIC, $\sigma_{y}=1 \mathrm{~nm}$ (and $40 \mathrm{~nm}$ in the horizontal direction). Additionally, as the bunch separation is only $0.5 \mathrm{~ns}$ (instead of $176 \mathrm{~ns}$ for ILC), the intra-pulse feedback is less effective. As a consequence, the permissible beam jitter is as low as $0.15 \mathrm{~nm}$ at $4 \mathrm{~Hz}$.

During the last two decades, several strategies to control the final focus have been investigated, and studied [5,7,11-15]. However, the performances of these strategies have not yet been objectively compared with a simple model, using realistic disturbances. In this paper, such a model is proposed, and updated using vibration spectra measured in the CMS experimental area of the LHC, which was identified as an environment representative of the final focus of a future linear particle collider. In the next 
section, we provide general considerations on final focus systems and on the opto-mechatronic approach followed in this study. Section 3 presents the simplified model of the final focus. In Sections 4-9, various control strategies are systematically tested and discussed. Section 10 summarizes the results, draws the conclusions and discusses the future work.

\section{Final focus}

The final focus of a particle collider is the part of the machine constituted of strong electromagnets, dedicated to focus the beams of particles to increase the density of the collisions. Each lattice of electromagnets ends with a pair of focusing (QF1) and defocusing (QD0) quadrupoles, which are often referred to as the final doublet. As there is one final doublet for each beam before the IP, the configuration of the magnet lattice near IP is typically QF1-QD0 IP QD0-QF1 (in principle, sextupoles are also added to reduce the chromaticity introduced by the quadrupoles). The capacity of the quadrupoles to produce a high luminosity depends on two factors: they must be extremely stable to avoid the jitters and sufficiently close to each other to maintain small beam crosssections. The first one requires innovative control strategies and will be extensively discussed in this paper. The second one is essentially the design parameter $L^{*}$, which is the distance between QD0 and the IP. As the size of the detector cannot be down-scaled, machine designers have two possibilities: either placing the final doublets at the end of the tunnel floor (i.e. large $L^{*}$, but a stable support) [16], or trying to insert them inside the detector (i.e. small $L^{*}$, but unstable floor). In this paper, only the latter case is considered, as the former one is much easier to control. For this latter case, several solutions have been proposed. For ILC, two configurations are currently studied in parallel: the Silicon Detector (SiD) [17] and the International Large Detector (ILD) [18]. In SiD, the last quadrupole (QD0) is supported by the endcap doors of the detector. In ILD, QD0 is supported by a huge beam, itself fixed at one end to a big pillar. Both detectors have been adapted for CLIC [19] and have been given the names CLICILD and CLIC-SiD. The QDO support structure will consist of a huge beam directly cantilevered to the tunnel wall. A possible simplified layout of this final focus is illustrated in Fig. 1.

To reach the required luminosity, two types of controllers are combined. The first one acts on the structure. Using vibration sensors (geophones, capacitive sensors, lasers), it tries to stabilize the quadrupoles. It works continuously. The second one acts on the particle beams. Using the measurement of the position of each pulse, it modifies the magnetic field applied to the next pulse with dipole correctors (kickers) to steer the beam and maintain a high collision luminosity. As there is only one pulse every $20 \mathrm{~ms}$, it works at $50 \mathrm{~Hz}$. A general block representation of the controllers is shown in Fig. 2. These two subsystems can be studied separately. However, in order to improve the performances of the design, the information contained in one subsystem can be used in the other subsystem, and conversely. For example, the beam control strategy can rely on the measurement from the geophone measuring the vibrations of the quadrupoles (feed forward in Fig. 2), or the information from the beam position monitor could be used to change the position of the quadrupole

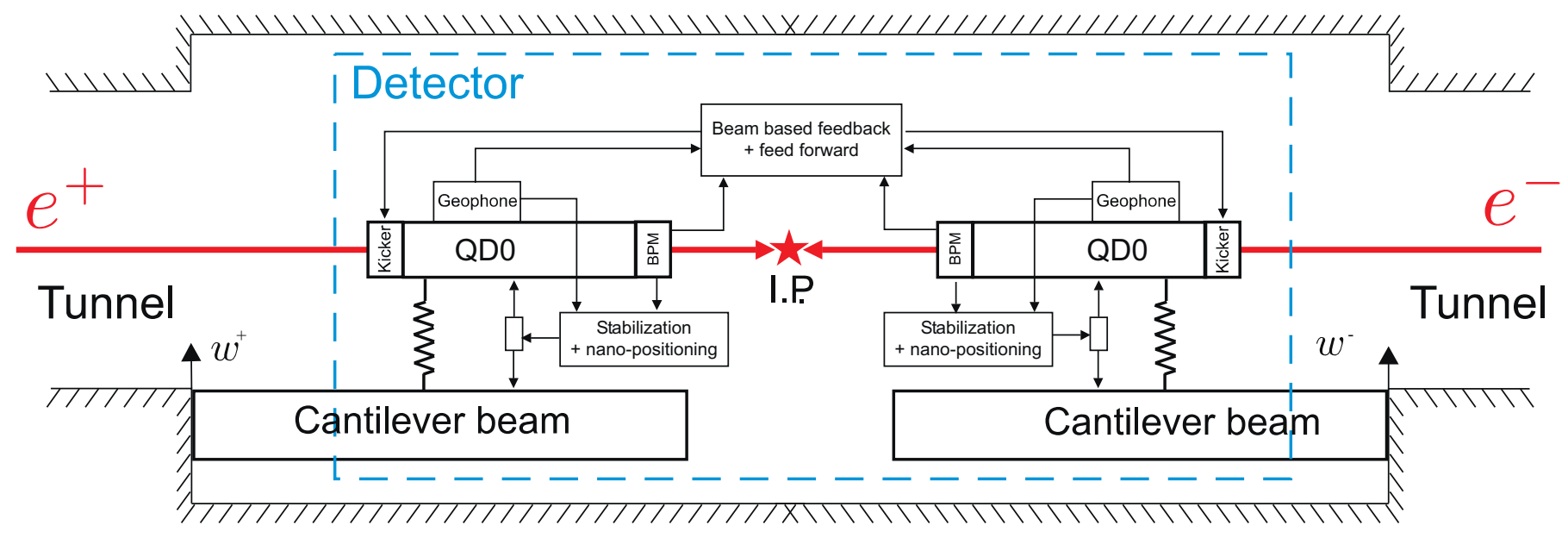

Fig. 1. Simplified layout of the final focus of a linear particle collider.

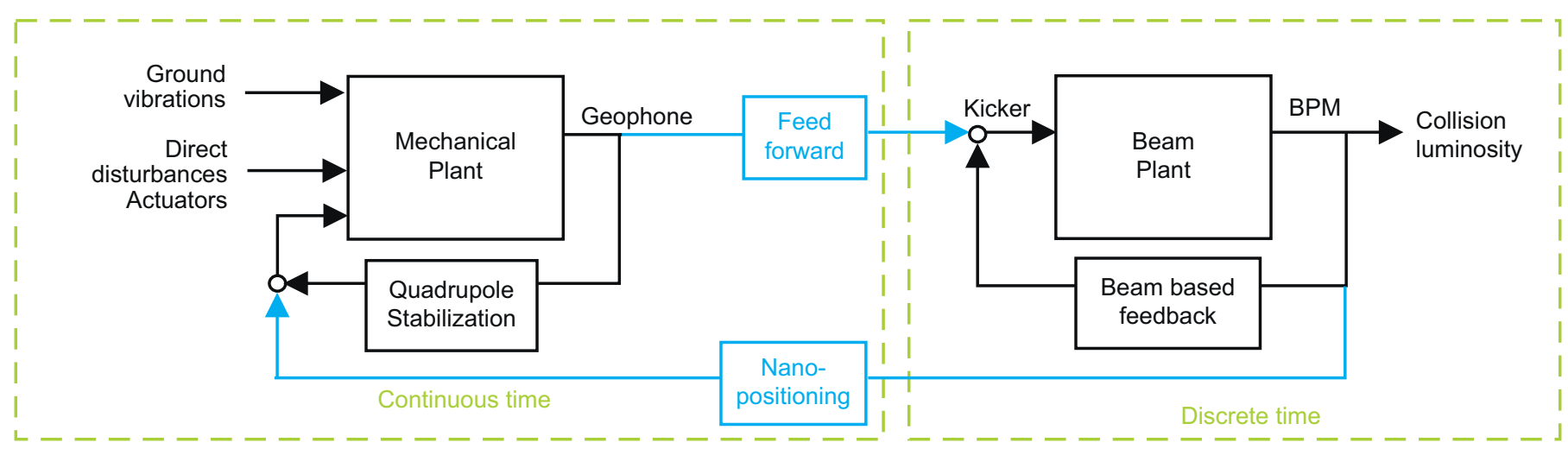

Fig. 2. Block diagram of the final focus hybrid system. 
(nanopositioning in Fig. 2). Adopting a holistic approach, the following strategies are reviewed or proposed ${ }^{1}$ :

- Pre-isolation of the whole structure.

- Damping of the structure with inertial feedback.

- Stiffening the structure with cables.

- Active stabilization on each side, using local inertial sensor.

- Beam based feedback, using the BPM, and the kickers.

- Feedforward, using inertial sensors to control the beam.

Their main advantages and limitations are discussed, and their compatibility/complementarity is established.

\section{Simplified model}

\subsection{Description of the system}

The model considered in this study, shown in Fig. 3, represents the system shown in Fig. 1, where only the vertical dynamics is taken into account. On each side of the IP, the mechanical system is represented by a three degrees of freedom (d.o.f.) system. From bottom to top, the huge cantilever beam is modelled as a single d.o.f. oscillator with a mass $m_{r}$, the girder is represented as an oscillator with a mass $m_{g}$, and the quadrupole is also modelled as a single d.o.f. oscillator with a mass $m_{q}$, fixed on the girder through an active mechanical stabilization support. Of course, if there is no girder, $m_{g}$ can also be seen as a second d.o.f. of the cantillever.

After the collision, the vertical displacement of the two particle beams, $y^{+}$and $y^{-}$, are measured by post-collision BPM. In Fig. 3, the analogy with optics has been used: the kickers are represented by prisms and the quadrupoles are represented by lenses. On each side of the IP, the BPM and kickers are attached to the quadrupole. In practice, the distance $L^{*}$ corresponds also the focal length of QD0, in such a way that the IP is located at the focal point of each QD0. Thus, a vertical displacement of the quadrupole $x_{q}$ induces a vertical displacement of the beam at the IP $y$.

The equations of the system are

$$
\left\{\begin{array}{l}
y^{+}=k^{+} x_{q}^{+} \\
y^{-}=k^{-} x_{q}^{-} \\
m_{q} \ddot{x}_{q}^{+}+k_{q}\left(x_{q}^{+}-x_{g}^{+}\right)+c_{q}\left(\dot{x}_{q}^{+}-\dot{x}_{g}^{+}\right)=f^{+} \\
m_{g} \ddot{x}_{g}^{+}+k_{g}\left(x_{g}^{+}-x_{r}^{+}\right)+c_{g}\left(\dot{x}_{g}^{+}-\dot{x}_{r}^{+}\right)+k_{q}\left(x_{g}^{+}-x_{q}^{+}\right)+c_{q}\left(\dot{x}_{g}^{+}-\dot{x}_{q}^{+}\right)=F^{+}-f^{+} \\
m_{r} \ddot{x}_{r}^{+}+k_{r}\left(x_{r}^{+}-w^{+}\right)+c_{r}\left(\dot{x}_{r}^{+}-\dot{w}^{+}\right)+k_{g}\left(x_{r}^{+}-x_{g}^{+}\right)+c_{g}\left(\dot{x}_{r}^{+}-\dot{x}_{g}^{+}\right)=0 \\
m_{q} \ddot{x}_{q}^{-}+k_{q}\left(x_{q}^{-}-x_{g}^{-}\right)+c_{q}\left(\dot{x}_{q}^{-}-\dot{x}_{g}^{-}\right)=f^{-} \\
m_{g} \ddot{x}_{g}^{-}+k_{g}\left(x_{g}^{-}-x_{r}^{-}\right)+c_{g}\left(\dot{x}_{g}^{-}-\dot{x}_{r}^{-}\right)+k_{q}\left(x_{g}^{-}-x_{q}^{-}\right)+c_{q}\left(\dot{x}_{g}^{-}-\dot{x}_{q}^{-}\right)=F^{-}-f^{-} \\
m_{r} \ddot{x}_{r}^{-}+k_{r}\left(x_{r}^{-}-w^{-}\right)+c_{r}\left(\dot{x}_{r}^{-}-\dot{w}^{-}\right)+k_{g}\left(x_{r}^{-}-x_{g}^{-}\right)+c_{g}\left(\dot{x}_{r}^{-}-\dot{x}_{g}^{-}\right)=0
\end{array}\right.
$$

The first two lines of Eq. (3) correspond to the beam vertical dynamics; the other lines correspond to the equations of the structure. ${ }^{2} x_{q}$ is the vertical displacement of the quadrupole, $x_{g}$ is the vertical displacement of the girder, and $x_{r}$ is the vertical displacement of the cantillever. All the masses are connected by springs $k_{r}, k_{g}, k_{q}$ as shown in Fig. 3. Small dashpots $\left(c_{r}, c_{g}, c_{q}\right)$ are also placed in parallel with the springs to add some damping, but are not shown for the clarity of the figure. The superscript '+' refers to the half part of the machine from which positrons arrive $\left(e^{+}\right)$, and the superscript '-' refers to the other half part of the

\footnotetext{
${ }^{1}$ The intra-pulse feedback, which tries to recover the luminosity inside each pulse [10], is out of the scope of this paper, and will not be discussed

2 Throughout all the paper, $y$ will always refer to a vertical beam displacement and $x$ to a vertical structural element displacement.
}

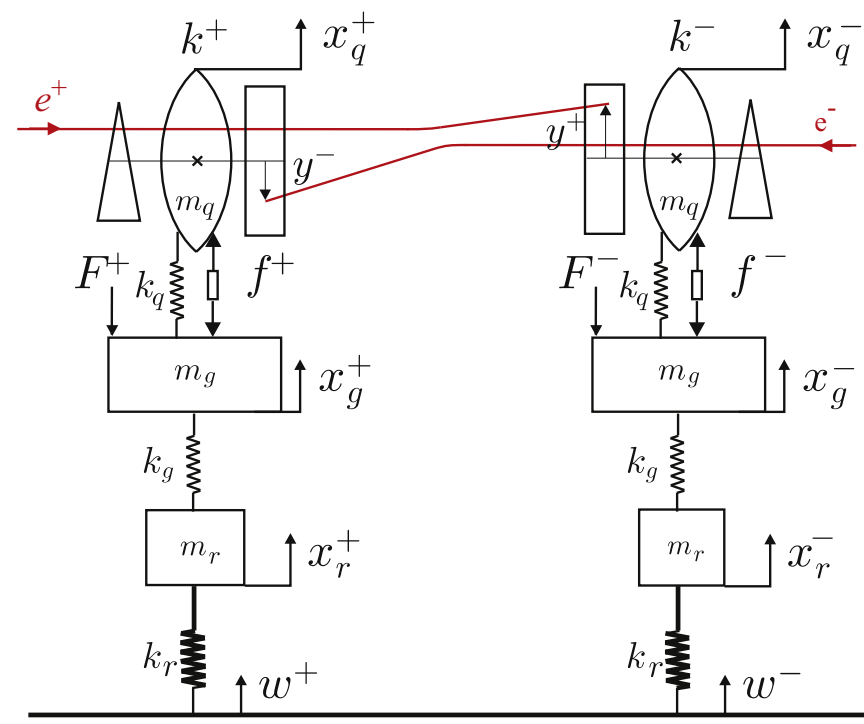

Fig. 3. Simplified model of the final focus

machine from which electrons arrive $\left(e^{-}\right) \cdot k^{+}$and $k^{-}$are the dimensionless parameters [20-22], taken as unity for simplicity.

In this simple approach, the unmodelled dynamics and the technical noise are represented by additional random forces $F^{+}$ and $F^{-}$, applied on the girder. Neglecting for now the first two lines, Eq. (3) can be written in a matrix form

$\mathbf{M} \ddot{\mathbf{x}}+\mathbf{C} \dot{\mathbf{x}}+\mathbf{K x}=\mathbf{B u}+\mathbf{L}^{\mathbf{F}} \mathbf{F}+k_{r} \mathbf{L}^{\mathbf{w}} \mathbf{w}$

where $\mathbf{X}=\left(x_{q}^{+}, x_{g}^{+}, x_{r}^{+}, x_{q}^{-}, x_{g}^{-}, x_{r}^{-}\right)^{T}$ is the vector of the degrees of freedom of the system, $\mathbf{u}=\left(f^{+}, f^{-}\right)^{T}$ is the vector of control forces, $\mathbf{F}=\left(F^{+}, F^{-}\right)^{T}$ is the vector of disturbing forces, $\mathbf{w}=\left(w^{+}, w^{-}\right)^{T}$ is the vector of ground excitations, $\mathbf{M}=\operatorname{diag}\left(m_{q}, m_{g}, m_{r}, m_{q}, m_{g}, m_{r}\right)$,

$\mathbf{K}=\left(\begin{array}{cc}\mathbf{K}^{+} & 0 \\ 0 & \mathbf{K}^{-}\end{array}\right) ; \quad \mathbf{C}=\left(\begin{array}{cc}\mathbf{C}^{+} & 0 \\ 0 & \mathbf{C}^{-}\end{array}\right)$

$\mathbf{K}^{+}=\left(\begin{array}{ccc}k_{q} & -k_{q} & 0 \\ -k_{q} & k_{g}+k_{q} & -k_{g} \\ 0 & -k_{g} & k_{g}+k_{r}\end{array}\right)=\mathbf{K}^{-}$

$\mathbf{C}^{+}=\left(\begin{array}{ccc}c_{q} & -c_{q} & 0 \\ -c_{q} & c_{g}+c_{q} & -c_{g} \\ 0 & -c_{g} & c_{g}+c_{r}\end{array}\right)=\mathbf{C}^{-}$

and the influence matrices are

$\mathbf{B}=\left(\begin{array}{cccccc}1 & -1 & 0 & 0 & 0 & 0 \\ 0 & 0 & 0 & 1 & -1 & 0\end{array}\right)^{T}$

$\mathbf{L}^{\mathbf{F}}=\left(\begin{array}{cccccc}0 & 1 & 0 & 0 & 0 & 0 \\ 0 & 0 & 0 & 0 & 1 & 0\end{array}\right)^{T}$

$\mathbf{L}^{\mathbf{w}}=\left(\begin{array}{llllll}0 & 0 & 1 & 0 & 0 & 0 \\ 0 & 0 & 0 & 0 & 0 & 1\end{array}\right)^{T}$

The numerical values have been chosen in such a way that the cantillever beam has roughly the same dynamics as the rotating shielding of the CMS experiment (Fig. 4): $m_{q}=400 \mathrm{~kg}$; $m_{g}=10^{3} \mathrm{~kg} ; m_{r}=10^{4} \mathrm{~kg} ; k_{q}=1.6 \times 10^{6} \mathrm{~N} / \mathrm{m} ; k_{g}=8 \times 10^{5} \mathrm{~N} / \mathrm{m} ;$ $k_{r}=2.5 \times 10^{6} \mathrm{~N} / \mathrm{m}$. Additionally, as metallic structures have a few percents of damping, the coefficients of the damping matrix $\mathbf{C}$ have been reasonably fixed as follows: $c_{q}=2 \times 0.01 \sqrt{k_{q} m_{q}}$; $c_{g}=2 \times 0.01 \sqrt{k_{g} m_{g}} ; c_{r}=2 \times 0.02 \sqrt{k_{r} m_{r}}$. 


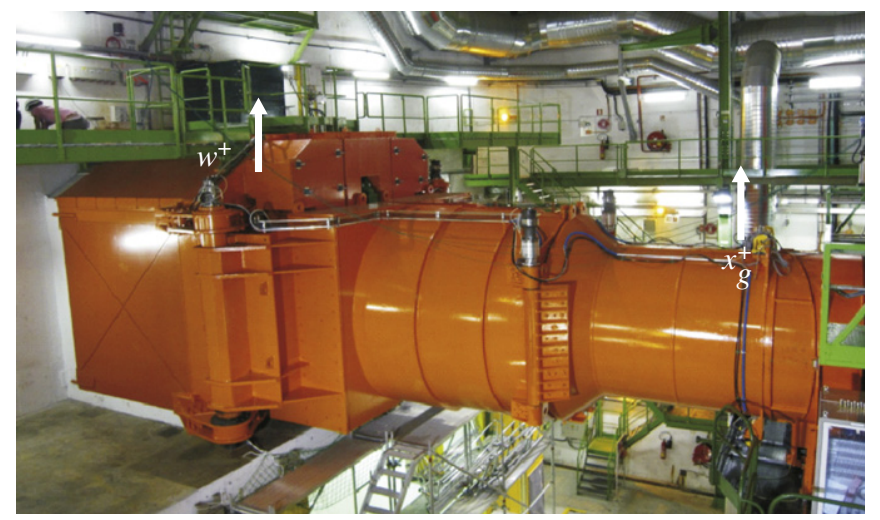

Fig. 4. Picture of the rotating shielding of the CMS experiment.
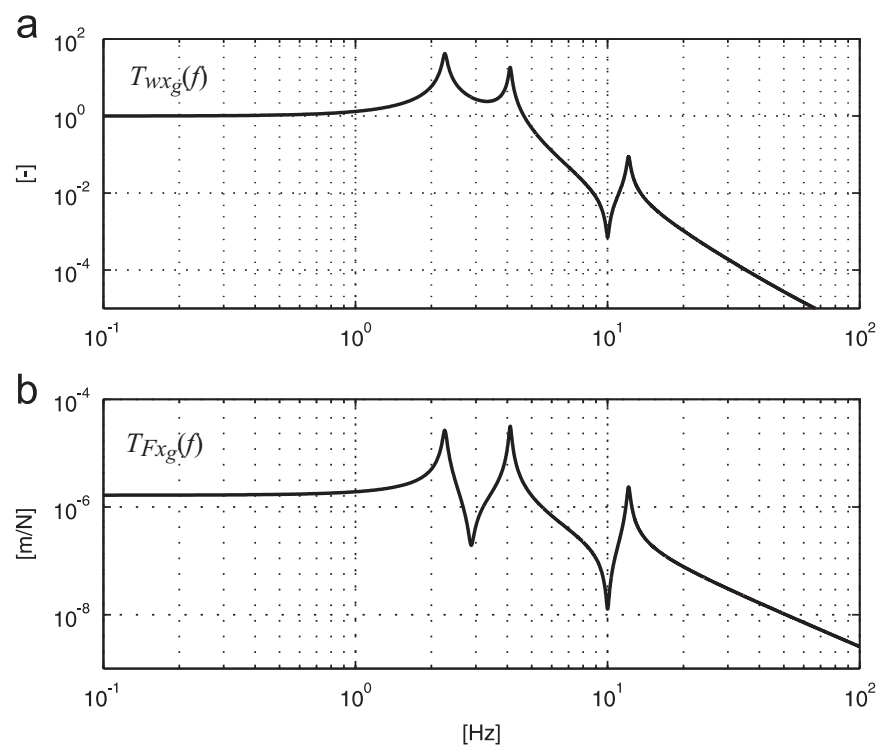

Fig. 5. (a) Transmissibility $T_{w x_{g}}$ between the ground and the girder support and (b) transmissibility $T_{w x_{g}}$ between the force $F$ applied on the girder and its displacement.

In the Laplace domain, Eq. (4) can be written as

$$
\mathbf{X}=\mathbf{G}\left(\mathbf{B u}+\mathbf{L}^{\mathbf{F}} \mathbf{F}+k_{r} \mathbf{L}^{\mathbf{w}} \mathbf{w}\right)
$$

where $\mathbf{G}$ is the plant of the system, given by

$\mathbf{G}=\left(\mathbf{M} s^{2}+\mathbf{C} s+\mathbf{K}\right)^{-1}$

and $s$ is the Laplace variable. Defining $\mathbf{T}_{\mathbf{u x}}=\mathbf{G B}$ as the transfer matrix between the control forces and the system coordinates, $\mathbf{T}_{\mathbf{F x}}=\mathbf{G L}^{\mathbf{F}}$ as the transfer matrix between the vector of forces applied on the system and its coordinates, and $\mathbf{T}_{\mathbf{w x}}(s)=\mathbf{G} k_{r} \mathbf{L}^{\mathbf{w}}$ as the transfer matrix between the support displacement and the system coordinates, Eq. (5) can be rewritten in compact form

$\mathbf{X}(s)=\mathbf{T}_{\mathbf{u x}}(s) \mathbf{U}(s)+\mathbf{T}_{\mathbf{w x}}(s) \mathbf{W}(s)+\mathbf{T}_{\mathbf{F x}}(s) \mathbf{F}(s)$

In Eq. (7), each element $\mathbf{T}_{\mathbf{A B}}(i, j)$ of the matrix $\mathbf{T}_{\mathbf{A B}}$ represents the transmission coefficient between $i$ th element of the source vector $\mathbf{A}$ and the $j$ th element of the response vector B. For example, in open loop, the transmissibility $T_{w x_{g}}$ between the ground and the girder support and the transmissibility $T_{\mathrm{Fx}_{\mathrm{g}}}$ between the force $F$ applied on the girder and its displacement are respectively shown in Fig. 5(a) and (b). The first two peaks are essentially the resonances of the two lower masses (vibrating in phase for the first mode, and out of phase for the second mode), while the third peak corresponds to the resonance of the quadrupole.

Referring to Eq. (3), the vertical relative displacement between the two colliding beams is given by

$\Delta y=\mathbf{R X}$

where $\mathbf{R}=\left(k^{+} 00-k^{-} 00\right)$ is the performance metrics vector.

\subsection{Error budgeting and calibration}

In this section, we evaluate formally how the imperfections are transmitted in the system. This procedure is known as the dynamic error budgeting. Three types of imperfections are considered: instrumentation noise, ground vibrations and technical noise. Assuming that these three noise sources are uncorrelated random processes, we can calculate the total error budgeting from (5) as

$\mathbf{S}_{\mathbf{X}}=\mathbf{G B S}_{\mathbf{u}}(\mathbf{G B})^{*}+\mathbf{G L}^{\mathbf{F}} \mathbf{S}_{\mathbf{F}}\left(\mathbf{G L}^{\mathbf{F}}\right)^{*}+\mathbf{G L}^{\mathbf{W}} \mathbf{S}_{\mathbf{W}}\left(\mathbf{G L}^{\mathbf{W}}\right)^{*}$

where $\mathbf{S}_{\mathbf{u}}, \mathbf{S}_{\mathbf{F}}$ and $\mathbf{S}_{\mathbf{w}}$ are the excitation matrices, defined as follows:

$\mathbf{S}_{\mathbf{u}}=\left(\begin{array}{cc}\Phi_{f^{+}} & 0 \\ 0 & \Phi_{f^{-}}\end{array}\right)$

$\mathbf{S}_{\mathbf{F}}=\left(\begin{array}{cc}\Phi_{F^{+}} & 0 \\ 0 & \Phi_{F^{-}}\end{array}\right)$

$\mathbf{S}_{\mathbf{w}}=\left(\begin{array}{cc}\Phi_{w^{+}} & \Phi_{w^{+} w^{-}} \\ \Phi_{w^{+} w^{-}} & \Phi_{w^{-}}\end{array}\right)$

In the matrices defined above, $\Phi$ denotes the Power Spectral Density (PSD) of the quantity in the subscript. Explicitly, the PSD of a signal $x(t)$ is defined as

$\Phi_{w^{+}}(f)=\int_{-\infty}^{\infty} A_{w^{+}}(\tau) e^{-i 2 \pi f \tau} d \tau$

where $A_{w^{+}}(\tau)$ is the auto-correlation function, defined by

$A_{w^{+}}(\tau)=\int_{-\infty}^{\infty} w^{+}(t) w^{+}(t+\tau) d t$

Similarly, $\Phi_{w^{+} w^{-}}$is the cross power spectral density between $w^{+}$and $w^{-}$is defined as the Fourier transform of the correlation function between the two signals, $A_{w^{+} w^{-}}(\tau)$, defined as

$A_{w^{+} w^{-}}(\tau)=\int_{-\infty}^{\infty} w^{+}(t) w^{-}(t+\tau) d t$

and ${ }^{*}$ stands for the conjugate transposed. $\mathbf{S}_{\mathbf{X}}$ is the response matrix, in which (i,j) element is $\Phi_{\mathbf{X}(i) \mathbf{X}(j)}$. Using compact notations

$\mathbf{S}_{\mathbf{X}}(f)=\left|\mathbf{T}_{\mathbf{u x}}(f)\right|^{2} \mathbf{S}_{\mathbf{u}}(f)+\left|\mathbf{T}_{\mathbf{w x}}(f)\right|^{2} \mathbf{S}_{\mathbf{w}}(f)+\left|\mathbf{T}_{\mathbf{F x}}(f)\right|^{2} \mathbf{S}_{\mathbf{F}}(f)$

and the power spectral density of the beam offset is given by

$\Phi_{\Delta}(f)=\mathbf{R} \mathbf{S}_{\mathbf{X}}(f) \mathbf{R}^{*}$.

The three terms on the right hand side of Eq. (13) are the three types of imperfections. The first term is the control noise, combining the sensor spectral noise transmitted through the control operation, the ADC and DAC noises, and the noise inherent to the actuator. All of these sources create parasitic forces applied to the system, whose power spectral densities (PSD) are respectively $\Phi_{f^{+}}$and $\Phi_{f^{-}}$. Actually, the dominant contribution in these forces is the sensor noise. From the computations [23], it appeared that for the seismometers used in this paper, the PSD of the sensor noise can be modelled as

$\Phi_{n}(f)=a f^{-5}+b N_{0}$

where we take $a=10^{-18}, b=2 \times 10^{-4}$ and $N_{0}=2 \times 10^{-25}$. The evaluation of the first term of Eq. (13) depends on the type of controller used, and will be discussed in Sections 6 and 8. The last 


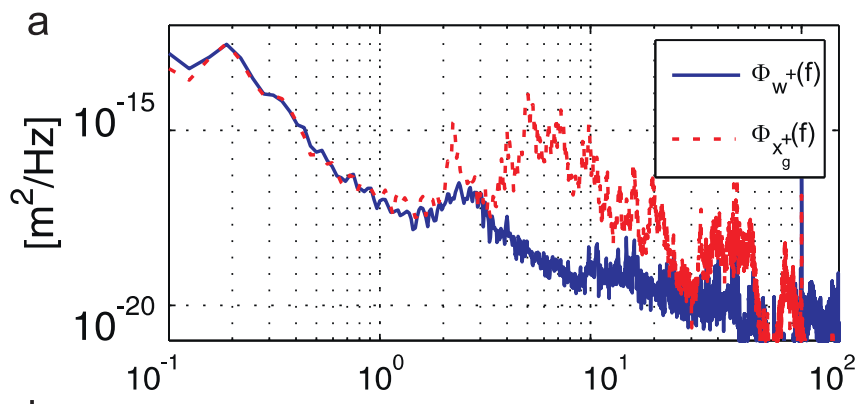

b
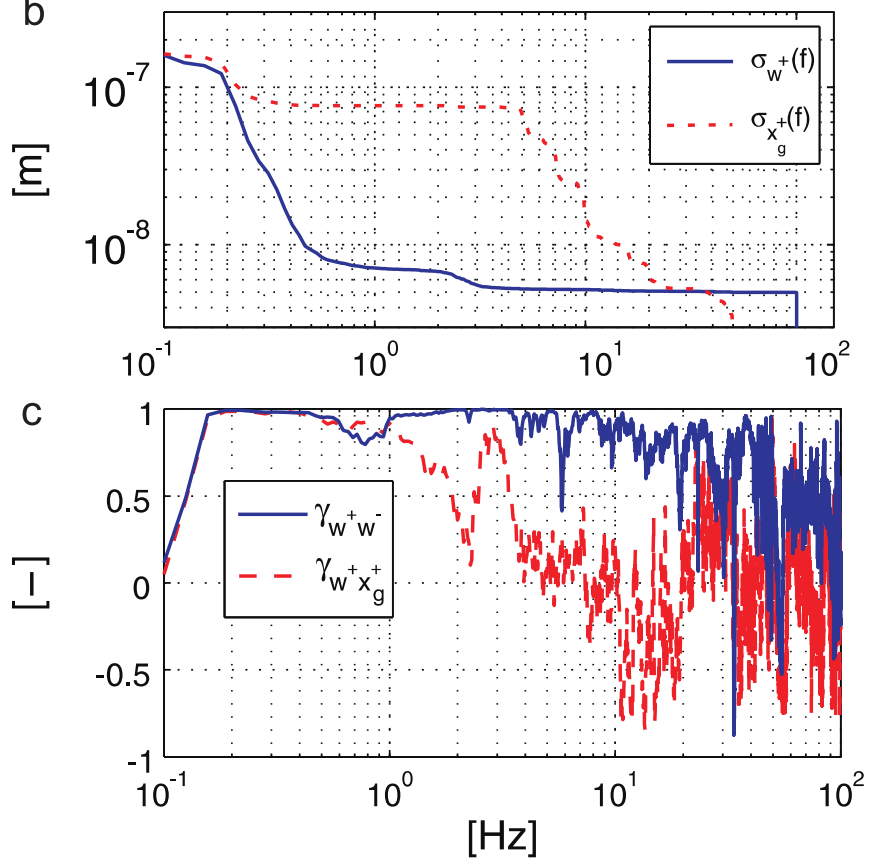

Fig. 6. Vibration measurements in the CMS experiment: (a) $\Phi_{w^{+}}$and $\Phi_{x_{g}^{+}}$; (b) $\sigma_{w^{+}}(f)$ and $\sigma_{x_{g}^{+}}(f)$; and (c) correlation $\gamma_{w^{+} w^{-}}(f)$ and $\gamma_{w^{+} x_{g}^{+}}(f)$.

two terms have been evaluated from vibration measurements in the CMS experiment [24]. For the second one, two seismometers have been placed on the ground floor, $6 \mathrm{~m}$ apart. Fig. 6(a) shows the PSD of only one signal, as it is identical to the other one. It is called $\Phi_{w^{+}}$in the adopted notations. Fig. 6(b) shows the corresponding integrated Root Mean Square (RMS) value, defined as

$\sigma_{w^{+}}(f)=\sqrt{\int_{f}^{\infty} \Phi_{w^{+}}(f) d f}$.

Fig. 6 (c) shows the correlation between $w^{+}$and $w^{-}$, defined as

$\gamma_{w^{+} w^{-}}(f)=\operatorname{Re}\left\{\frac{\Phi_{w^{+} w^{-}}}{\sqrt{\Phi_{w^{+}} \Phi_{w^{-}}}}\right\}$

from which one can readily evaluate the second term of Eq. (13). Basically, $\gamma$ represents the causality between two signals: if $\gamma \simeq 1$, then the signals are correlated (proportionality relationship and in phase), if $\gamma \simeq-1$, then the signals are anti-correlated (proportionality relationship and out of phase), and if $\gamma \simeq 0$, then the signals are not correlated [25].

To evaluate the third term, a second measurement has been performed in CMS. One seismometer has been placed close to the base of the rotating shielding, measuring $w^{+}$has been calculated, and one seismometer has been placed at the end of the rotating shielding, measuring $x_{g}^{+}$. These measurements have been performed when the water cooling was turned off. The power spectral densities of these signals, $\Phi_{w^{+}}(f)$ and $\Phi_{x_{g}^{+}}(f)$, and the corresponding integrated RMS $\sigma_{w^{+}}(f)$ and $\sigma_{x_{g}^{+}}(f)$ are shown respectively in Fig. 6(a) and (b). Below $2 \mathrm{~Hz}$, i.e. below the first structural resonance, $\Phi_{w^{+}}(f)$ and $\Phi_{x_{+}^{+}}(f)$ are nearly identical, which means that the rotating shielding is essentially moving as a rigid body. However, above $2 \mathrm{~Hz}$, the vibrations at the end of the tube are much larger. Above $5 \mathrm{~Hz}, \Phi_{x_{g}^{+}}$is already around $80 \mathrm{~nm}$,
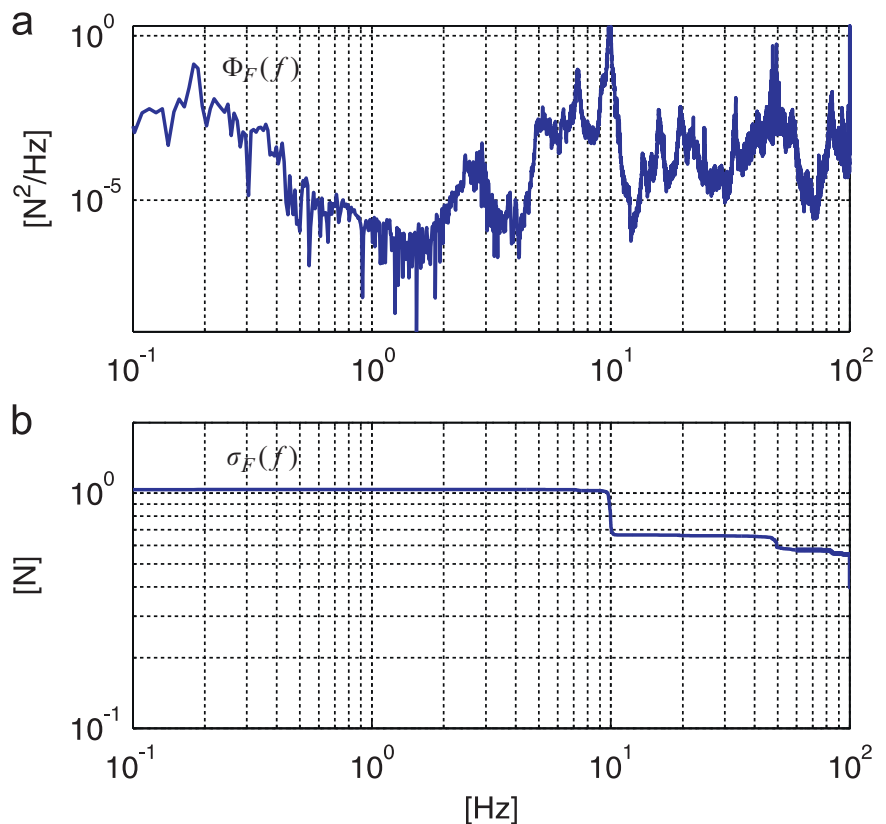

Fig. 7. (a) Power spectral density of the force $\Phi_{F}^{+}(f)$ calculated using Eq. (18) and (b) corresponding integrated RMS value of the force $\sigma_{F}(f)$
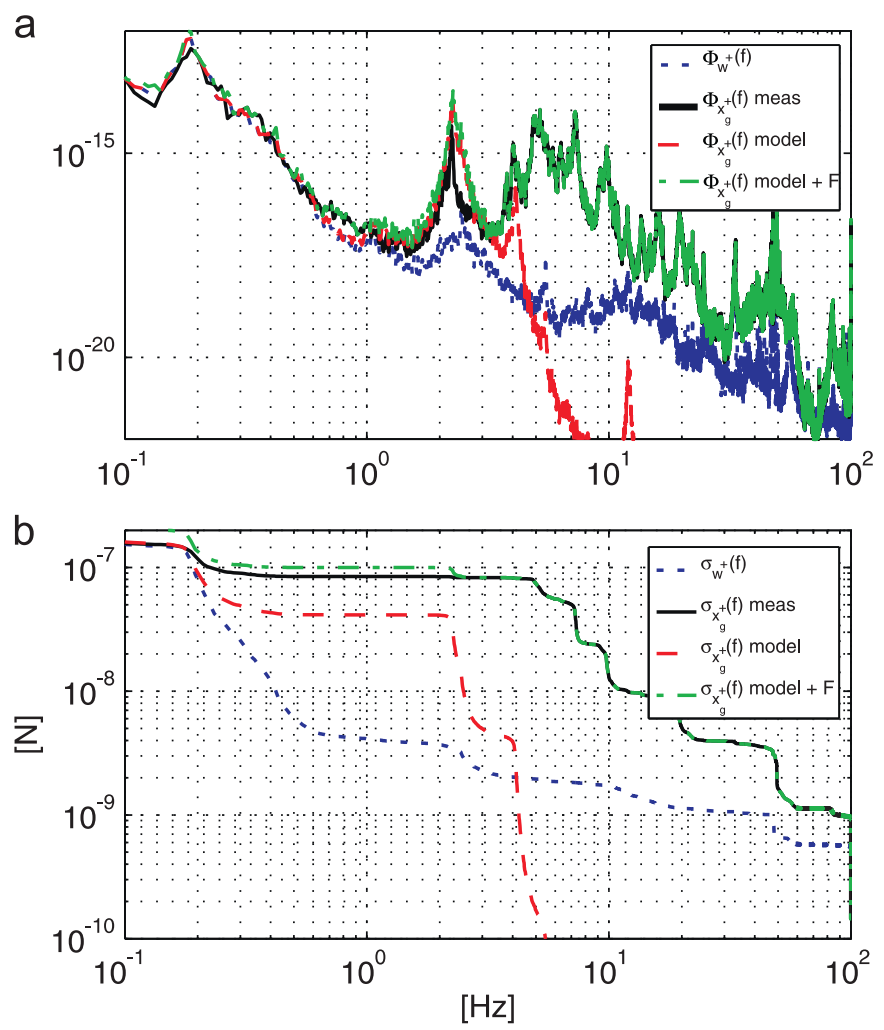

Fig. 8. Effect of $F^{+}$on the system response: (a) PSD and (b) integrated RMS of $w^{+}$ $x_{g}^{+}$measured, $x_{g}^{+}$calculated without $F^{+}$and $x_{g}^{+}$calculated with $F^{+}$. 
which is more than 500 times larger than the requirement for the vertical motion of the quadrupole. Actually, these large vibrations of the tube are caused by the technical noise, and not by the ground vibrations, as illustrated by $\gamma_{w^{+} x_{g}^{+}}$in Fig. 6(c). The curve is rapidly falling down to zero above $2 \mathrm{~Hz}$, showing that there is no causal relationship between the two signals. In the model, the technical noise is represented by a random force, $F^{+}$, applied on the cantillever, and is evaluated as follows.

In open loop, and considering only the left part of the final focus, Eq. (13) can be transformed into

$\Phi_{F^{+}}(f)=\left|T_{F^{+} x_{g}^{+}}(f)\right|^{-2}\left\{\Phi_{x_{g}^{+}}(f)-\left|T_{w^{+} x_{g}^{+}}(f)\right|^{2} \Phi_{w^{+}}(f)\right\}$

from which the third term of Eq. (13) can be determined, assuming that $\Phi_{F^{-}}(f)=\Phi_{F^{+}}(f)$, and no correlation between $F^{+}$ and $F^{-}$. The resulting PSD and integrated RMS of $F^{+}$are shown in Fig. 7(a) and (b).

It is interesting to note that the RMS value is as small as $1 \mathrm{~N}$. In order to validate the calculation, Fig. 8(a) and (b) shows respectively the PSD and integrated RMS of $w^{+}, x_{g}^{+}$measured, $x_{g}^{+}$ calculated without $F^{+}$and $x_{g}^{+}$calculated with $F^{+}$. One clearly sees that if $\mathrm{F}^{+}$is not considered, at $4 \mathrm{~Hz}$, the model underestimates the value of $x_{g}^{+}$by nearly two orders of magnitude.
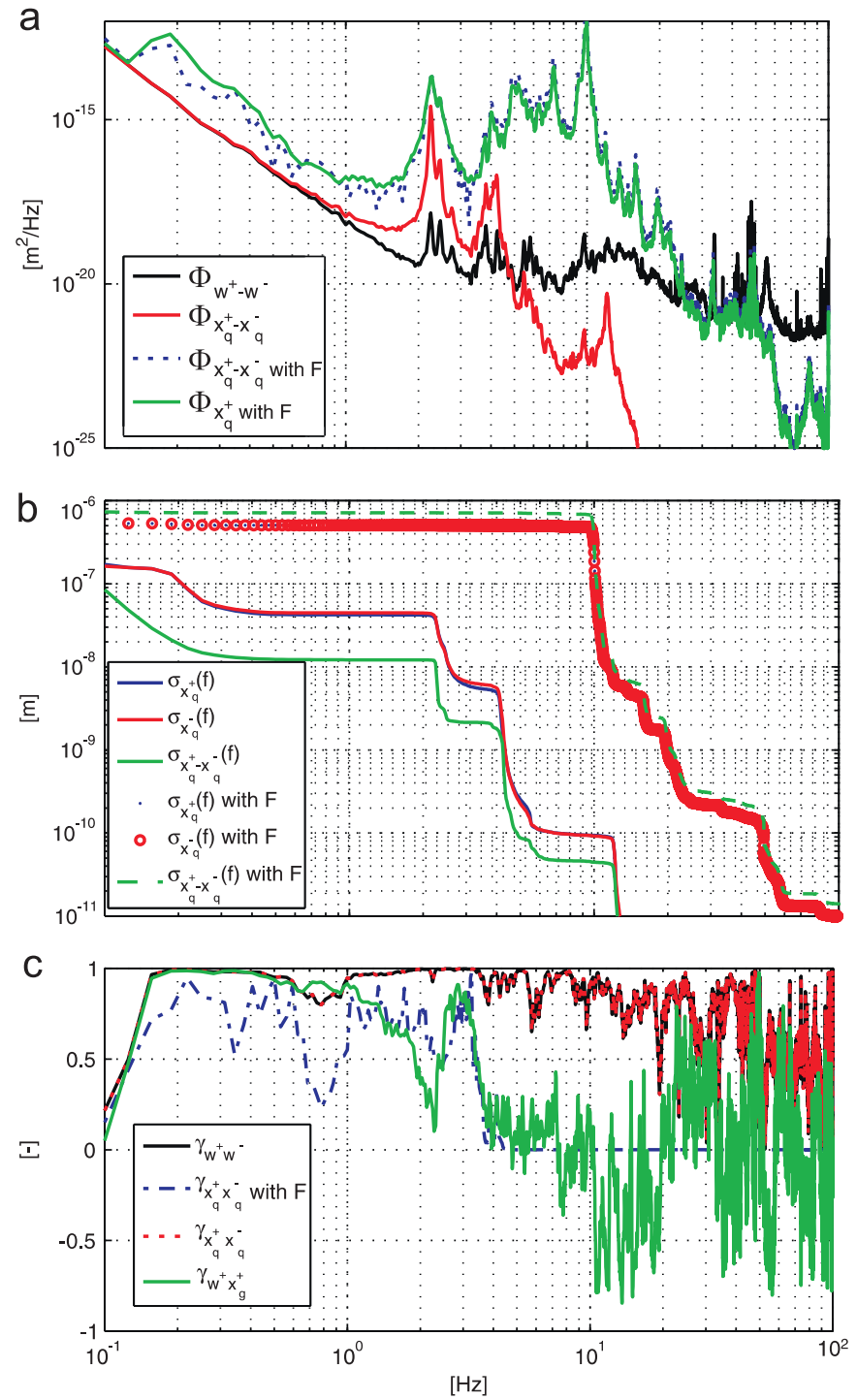

Fig. 9. Validation of the model. Comparison of predicted and measured: (a) PSDs, (b) integrated RMS, and (c) correlations.
On the other hand, when $F^{+}$is considered, the predicted and measured PSD of $x_{g}^{+}$are in very good agreement.

To complete the validation of the model, we now consider the whole system. Fig. 9(a)-(c) shows respectively the PSD, Integrated RMS, and correlation of relative displacements: $w^{+}-w^{-}, x_{q}^{+}-x_{q}^{-}$. One sees that when $\mathbf{F}$ is not considered, $\gamma_{x_{q}^{+} x_{q}^{-}}$is close to $\gamma_{w^{+} w^{-}}$; when $\mathbf{F}$ is considered, $\gamma_{x_{q}^{+} x_{q}^{-}}$very close to the measured $\gamma_{w^{+} x_{\bar{\sigma}}^{-}}$, which is a much more realistic assumption. The importance to consider $\mathbf{F}$ will clearly appear in the following sections.

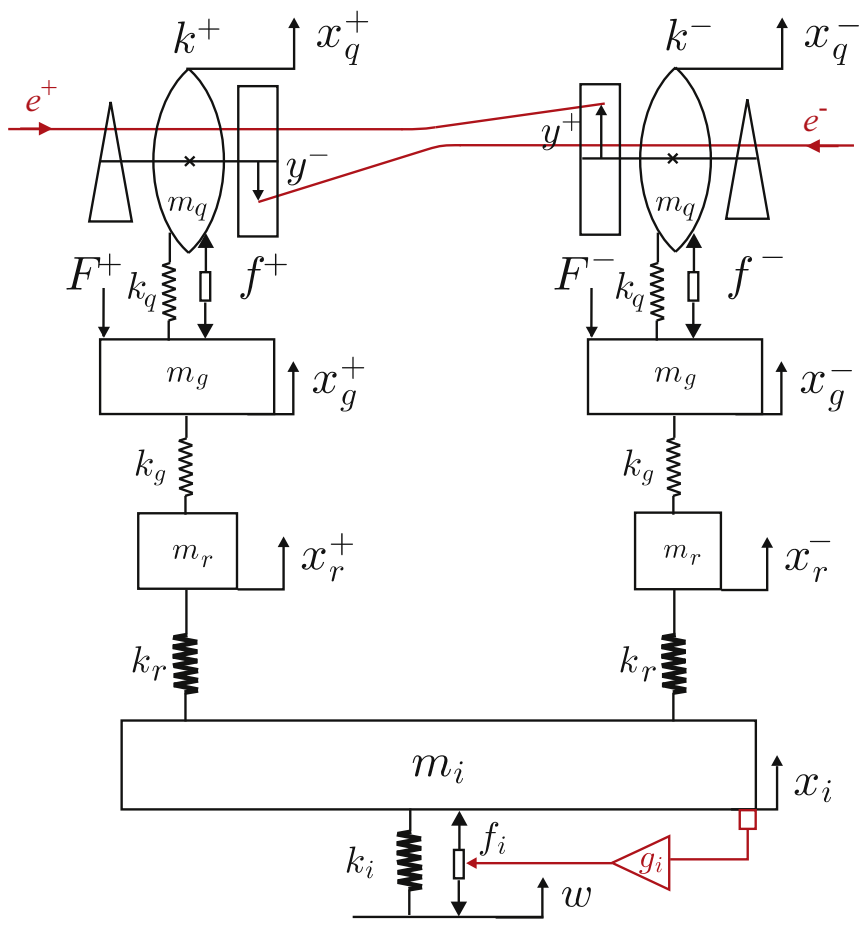

Fig. 10. Simple model of the pre-isolator.
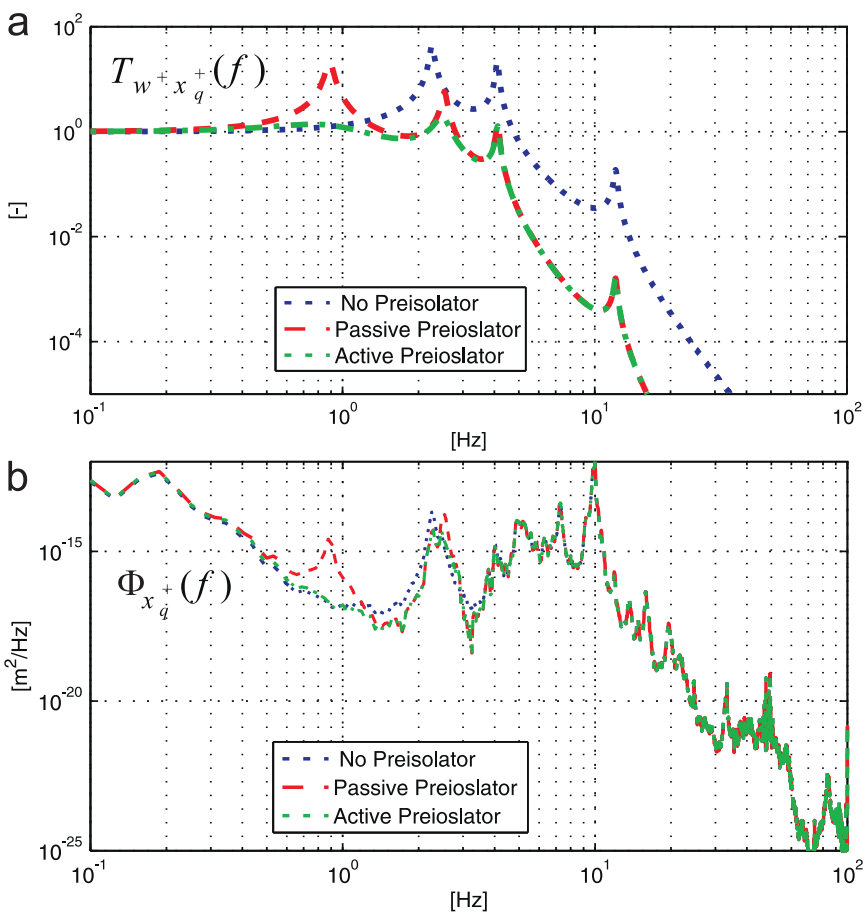

Fig. 11. Effect of a pre-isolator on the motion of the quadrupole: (a) transmissibility $T_{w+x_{q}^{+}}$between the ground and the quadrupole and (b) $\Phi_{x_{q}^{+}}$. 


\section{Pre-isolator}

In the previous section, it has been shown that a major disadvantage of the cantilevered configuration is that the vibrations at the end of the tube are much larger than close to the end of the tunnel (see Fig. 8).

To minimize this effect, an idea is to decouple the last quadrupoles of each line (QD0 and QF1) from the ground. To this purpose, the solution proposed is to mount these quadrupoles on a massive concrete slab (about 50-100 tons), itself mounted on air springs (Fig. 10) [26]. The resonance frequency of the concrete slab on such spring can be tuned around $1 \mathrm{~Hz}$.

Fig. 11(a) shows the transmissibility $T_{w^{+} x_{q}^{+}}$between the ground and the quadrupole. One sees that the passive pre-isolator has a clear effect of decreasing $T_{w^{+} x_{q}^{+}}$above its resonance frequency, at the cost of an amplification at its resonance. The overshoot can further be demagnified actively as follows. In parallel to the air spring, we place a soft actuator, e.g. a shielded Lorentz actuator (see Fig. 10). Provided that a signal proportional to the absolute velocity of the concrete slab can be measured at low frequency, and using a proportional control, the force $f_{i}$ delivered by the actuator is

$f_{i}=-g_{i} \dot{x}_{i}$

where $g_{i}$ is the gain of the controller. Using such a velocity feedback, often called a sky-hook damper [27], the resonance peak can be removed, without any degradation of the isolation at high frequency.

Such a solution has been already adopted at several places where a very stable vibrational environment is required, and thus could theoretically lead to a great improvement of the stability of QD0 and QF1. Fig. 11(b) shows the effect of the passive and active pre-isolator on the $\Phi_{x_{q}^{+}}$. One sees however that above $2 \mathrm{~Hz}$, none of the pre-isolators can reduce the vertical displacement of the quadrupole. Actually, this solution is based on the assumption that the vibrations at the end of the rotating shielding are caused by the vibrations of the floor, i.e. that $\gamma_{w^{+} x_{g}^{+}}=1$. However, we know from Fig. 6(c) that above $2 \mathrm{~Hz}$, there is no causal relationship between the vibrations of the floor, and the vibrations of the cantillever tip. As a conclusion, it is important to take the technical noise into account (third term of Eq. (13)) to make a realistic evaluation of the pre-isolator performances. Similarly,
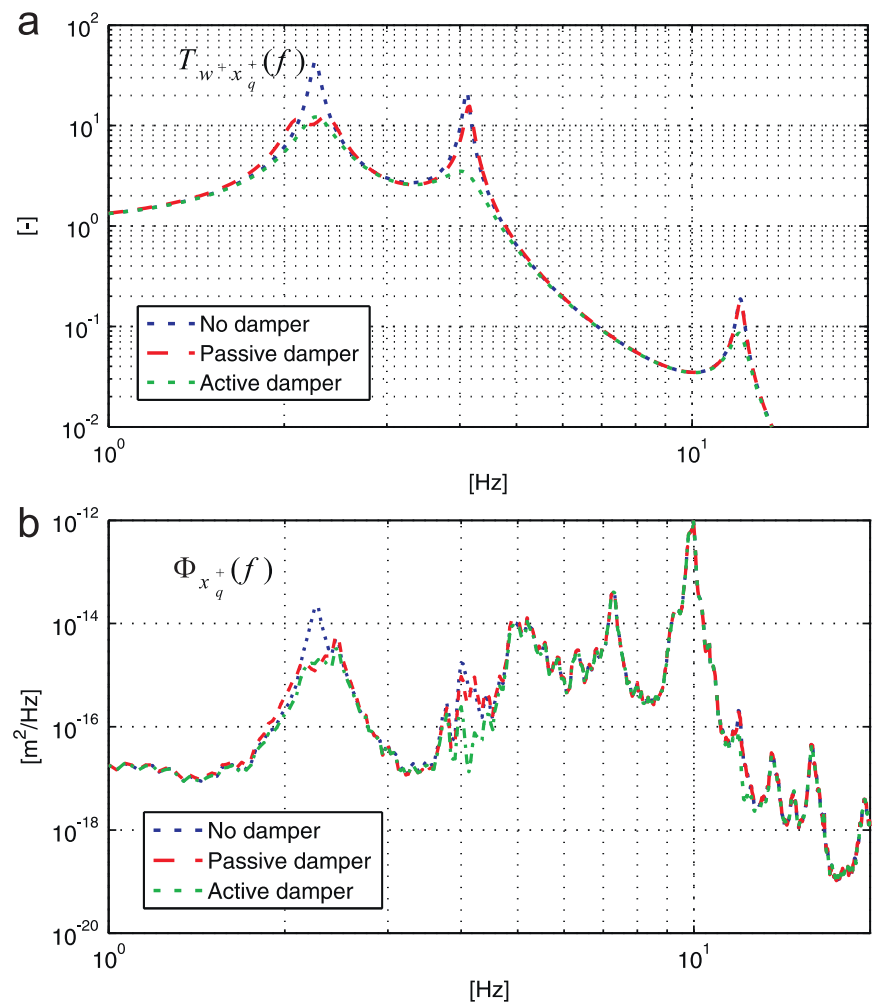

Fig. 13. Effect of vibration dampers on the motion of the quadrupole: (a) transmissibility $T_{w^{+} x_{q}^{+}}$between the ground and the quadrupole and (b) $\Phi_{x_{q}^{+}}$.
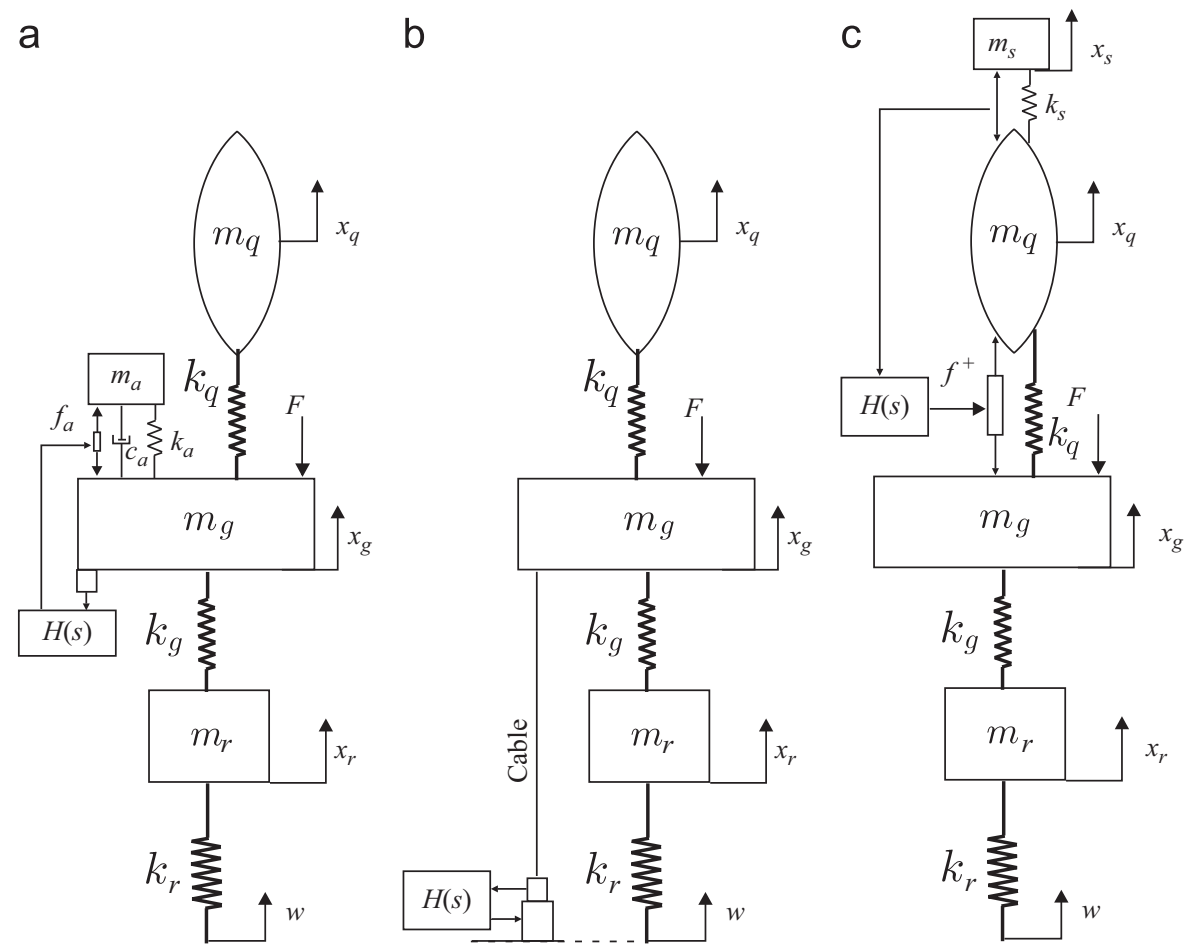

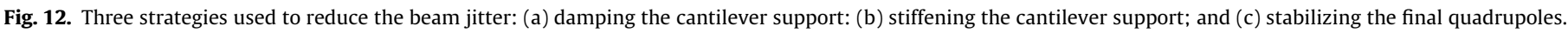


high frequency spurious modes can also substantially affect the isolation property of the pre-isolator [26].

\section{Damping the cantilever support}

In order to increase the stability of the final focus, one solution consists of damping the cantilever support. One solution consists of appending small oscillators on the structure, and tune their parameters on target modes of the structure. A possible configuration is shown in Fig. 12(a) for one half of the final focus, and in the absence of the controller. As an illustration, Fig. 13(a) shows the reduction in the transmissibility $T_{w^{+} x_{a}^{+}}(f)$ achieved with an oscillator tuned on the first mode, and $m_{a}=100 \mathrm{~kg}$. The major disadvantage of this method is that it requires as much oscillators as the number of structural resonances to damp, or multi-degreeof-freedom oscillators [28]. A solution to damp several resonances with a single device is provided by an active mass damper $[29,30]$, also shown in Fig. 12(a). In this case, however, the parameters are chosen such as $f_{A} \ll f_{1}$, where $f_{A}$ is the resonance frequency of the actuator, and $f_{1}$ is the first structural resonance, and the active mass damper works as a perfect force generator above $f_{A}$.

Using perfect velocity sensor and a simple proportional control $H(s)=g$, the force delivered by the actuator is

$f_{a}=-g \dot{x}_{g}^{+}$

The performances of the active mass damper are also shown in Fig. 13(a). One sees that higher order modes are also damped by the active mass damper. Fig. 13(b) shows $\Phi_{x_{q}^{+}}$for the two dampers. The amplitude of the motion is slightly reduced at the first resonance, but not elsewhere, and will obviously not lead to any significant reduction in the global motion of the quadrupole. Again, the disturbing forces are the root causes for the poor performances.

In order to increase the robustness of the system to technical noise, it is necessary to decrease its compliance, i.e. increase its dynamic stiffness, which is developed in the next section.

\section{Stiffening the support}

The solution proposed to stiffen the support consists of fixing active cables between the end of the cantilever tube and stable points. Ideally, the points are outside the detector, but the cables can also be attached to the solenoid cryostat, like the carbon fibre tie rods used to suspend the QD0 support structure in the ILD. One possible configuration is shown in Fig. 12(b) where, for each side, a single cable connects the cantilever to the ground. Because of their low natural damping, the cables are connected to an active tendon at one end, consisting of a displacement piezoelectric actuator in series with a force sensor. Neglecting the dynamics of the active cables, Eq. (4) becomes

$$
\mathbf{M} \ddot{\mathbf{x}}+\mathbf{C} \dot{\mathbf{x}}+\mathbf{K x}=\mathbf{B u}+\mathbf{L}^{\mathbf{F}}(\mathbf{F}+\mathbf{T})+k_{r} \mathbf{L}^{\mathbf{w}} \mathbf{w}
$$

where $\mathbf{T}=\left(T^{+}, T^{-}\right)^{T}$ is the vector of tensions in the active cables, given by

$$
\left(\begin{array}{c}
T^{+} \\
T^{-}
\end{array}\right)=k_{c}\left[\left(\begin{array}{c}
x_{q}^{+} \\
x_{q}^{-}
\end{array}\right)-\left(\begin{array}{c}
w^{+} \\
w^{-}
\end{array}\right)-\left(\begin{array}{c}
\delta^{+} \\
\delta^{-}
\end{array}\right)\right]
$$

where $k_{c}$ is the axial stiffness of the cables and $\delta^{+}$and $\delta^{-}$are the elongations of the piezoelectric actuators. Combining Eqs. (20) and (21), we get

$$
\mathbf{M} \ddot{\mathbf{x}}+\mathbf{C} \dot{\mathbf{x}}+\left[\mathbf{K}+k_{c} \mathbf{L}^{\mathbf{F}}\left(\mathbf{L}^{\mathbf{F}}\right)^{T}\right] \mathbf{x}=\mathbf{B u}+\mathbf{L}^{\mathbf{F}} \mathbf{F}+\left(k_{r} \mathbf{L}^{\mathbf{w}}+k_{c} \mathbf{L}^{\mathbf{F}}\right) \mathbf{w}
$$

This equation indicates that $\mathbf{K}+k_{c} \mathbf{L}^{\mathbf{F}}\left(\mathbf{L}^{\mathbf{F}}\right)^{T}$ is the new stiffness matrix of the structure, which has been increased by the stiffness
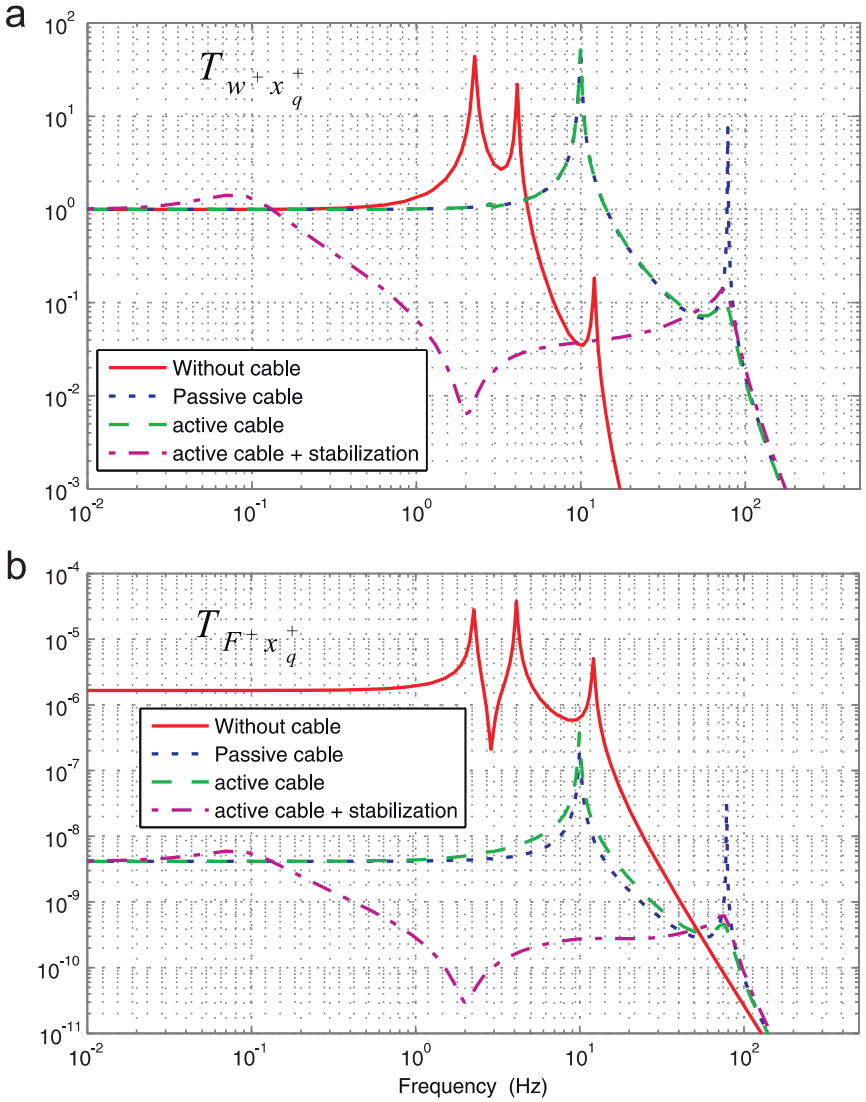

Fig. 14. (a) Transmissibility between the ground and the quadrupole and (b) compliance between $\mathrm{F}^{+}$and $x_{q}^{+}$.

of the cables. Then, the tension in the cables is measured by force sensors, and used to modify the elongations of the actuators according to

$\delta=g H(s) k_{c}^{-1} T$

where $g$ is a gain and $H(s)$ is the controller applied to the cable. In order to increase the damping without softening the system, the following controller is used [31]:

$H(s)=\frac{s}{(s+a)^{2}}$

where $a$ is a constant

Fig. 14(a) and (b) shows respectively the transmissibility $T_{w^{+} x_{q}^{+}}(f)$ and the compliance $T_{F^{+} x_{q}^{+}}(f)$ for both passive and active cables, and $k_{c}=240 \mathrm{MN} / \mathrm{m}$. One sees that above $5 \mathrm{~Hz}$ the isolation has been significantly reduced by the presence of the cables. However, at the same time, the compliance has been reduced by more than a factor 100 .

Fig. 15(a) and (b) shows the PSD and integrated RMS value of the relative displacement between the last two quadrupoles. Even though it is significantly decreased in a broad frequency range between $2 \mathrm{~Hz}$ and $30 \mathrm{~Hz}$, the RMS is only decreased by a factor 2 , essentially because of the high excitation at $10 \mathrm{~Hz}$. The active tendons remove the big overshoot at $80 \mathrm{~Hz}$, which also locally reduce the RMS value of the quadrupole relative displacement.

Apart from the increased robustness to the technical noise, the cables also allows to change the position of the cantilever tube, and can potentially replace the alignment stage currently foreseen inside the tube [32]. 

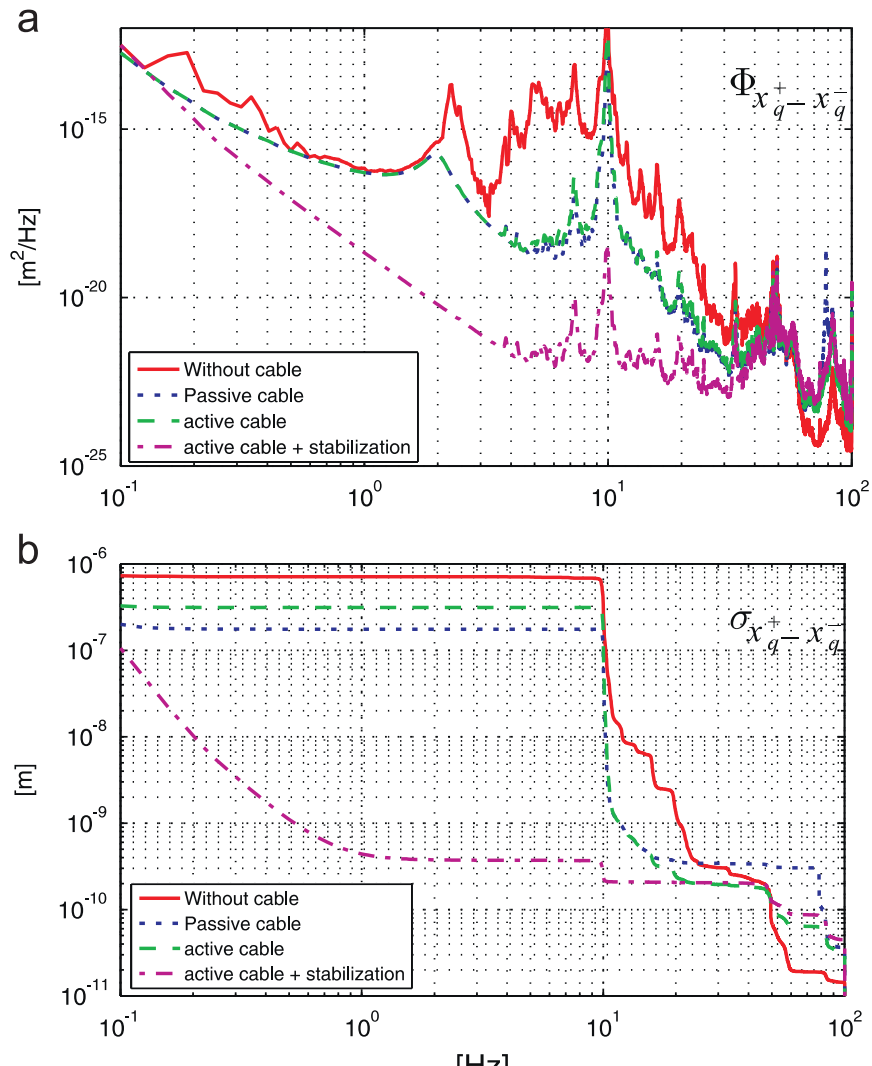

$[\mathrm{Hz}]$

Fig. 15. (a) Power spectral density of the relative quadrupole displacement $x_{q}^{+}-x_{q}^{-}$ and (b) integrated RMS value of the curves shown in (a).

\section{Active stabilization of the quadrupoles}

Several control strategies have been tested to stabilize actively the final doublets [29,33-35]. For example, in Refs. [33,34], the control force is proportional to the absolute velocity of the quadrupole. In Refs. [6,30], the control force is proportional to the relative displacement between the quadrupole and a reference mass. Another class of solutions consists of installing a network of interferometers, sometimes referred as optical anchor, to measure the distance between the final focus magnets and a reference point [16]. In this section, we will consider only the simple case of an absolute displacement feedback for each quadrupole. One half of the system is shown in Fig. 12(c). The control forces are given by

$f^{+}=g H(s) x_{q}^{+}, \quad f^{-}=g H(s) x_{q}^{-}$

where $g$ is the gain and $H(s)$ is the controller, including the sensor dynamics, a lag at $0.5 \mathrm{~Hz}$ to reduce the overshoot and a lead at $100 \mathrm{~Hz}$ to improve the stability. Again, the transmissibility $T_{w^{+} x^{+}}$ and the compliance $T_{F^{+} x_{a}^{+}}$are shown in Fig. 14(a) and (b). Both of them are reduced between $0.2 \mathrm{~Hz}$ and $30 \mathrm{~Hz}$, leading to a reduction in the RMS value of $x_{q}^{+}-x_{q}^{-}$limited essentially by the sensor noise, as shown in Fig. 15.

\section{Beam based feedback}

\subsection{Corrector dipoles}

The two beams are constituted of pulses containing the particles, arriving at the IP at a frequency called the repetition rate. For CLIC, the repetition rate is $50 \mathrm{~Hz}$. The basic principle of the beam-based feedback is to minimize the beam-beam offset, measured with the Beam Position Monitors (BPMs), by deflecting the beam with the corrector dipoles (Figs. 1 and 2). Then, by subtracting the first two lines of Eq. (3) and assuming for simplicity that $k^{+}=k^{-}=1$, we get

$\Delta y(n)=\Delta x(n)-\Delta b(n)$

which expresses that at the discrete time $n$, the beam-beam offset $\Delta y(n)$ is approximated by the relative vertical displacement between the quadrupoles $\Delta x(n)$, minus the compensation $\Delta b(n)$ imposed by the correctors

$\Delta b(n)=H(n)\left[\Delta y(n)+n_{y}(n)\right]$

where $H(n)$ is the controller and $n_{y}$ the BPM noise. Combining Eqs. (26) and (27) and using the Z-transform (because the signals are discrete), the beam offset is

$\Delta y(z)=\frac{1}{1+H(z)} \Delta x(z)-\frac{H(z)}{1+H(z)} n_{y}(z)$

To compensate for low frequency ground motion, a second order controller is necessary, which has the general form

$H(z)=\frac{a_{1}+a_{2} z^{-1}+a_{3} z^{-2}}{a_{4}+a_{5} z^{-1}+a_{6} z^{-2}}$.

Fig. 16(a) and (b) shows the PSD and integrated RMS of the beam-beam offset, using the following numerical values of the parameters in Eq. (29) [36]: $a_{1}=0 ; a_{2}=1 ; a_{3}=-0.5, a_{4}=1$; $a_{5}=-1.5 ; a_{6}=1$. The BPM noise has been assumed as a white noise with a RMS value of $20 \mathrm{pm}$.

One sees that good rejection at very low frequency is obtained at the cost of an amplification of the technical noise. In Ref. [37],
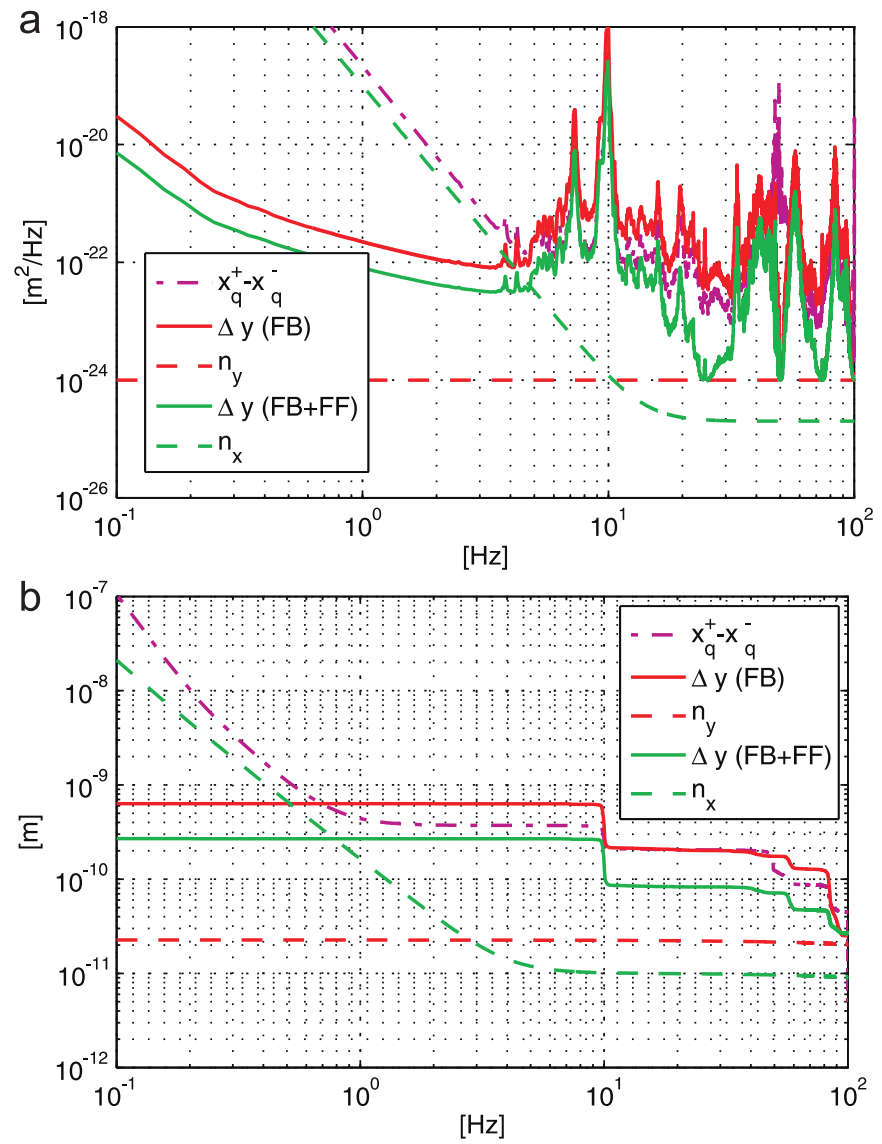

Fig. 16. (a) PSD and (b) integrated RMS of the residual motion $x_{q}^{+}-x_{q}^{-}$, the beambeam offset $\Delta y$ with feedback only (FB) and with both feedback and feed forward $(\mathrm{FB}+\mathrm{FF})$, the BPM noise $n_{y}$ and the vibration sensor noise $n_{x}$. 
a
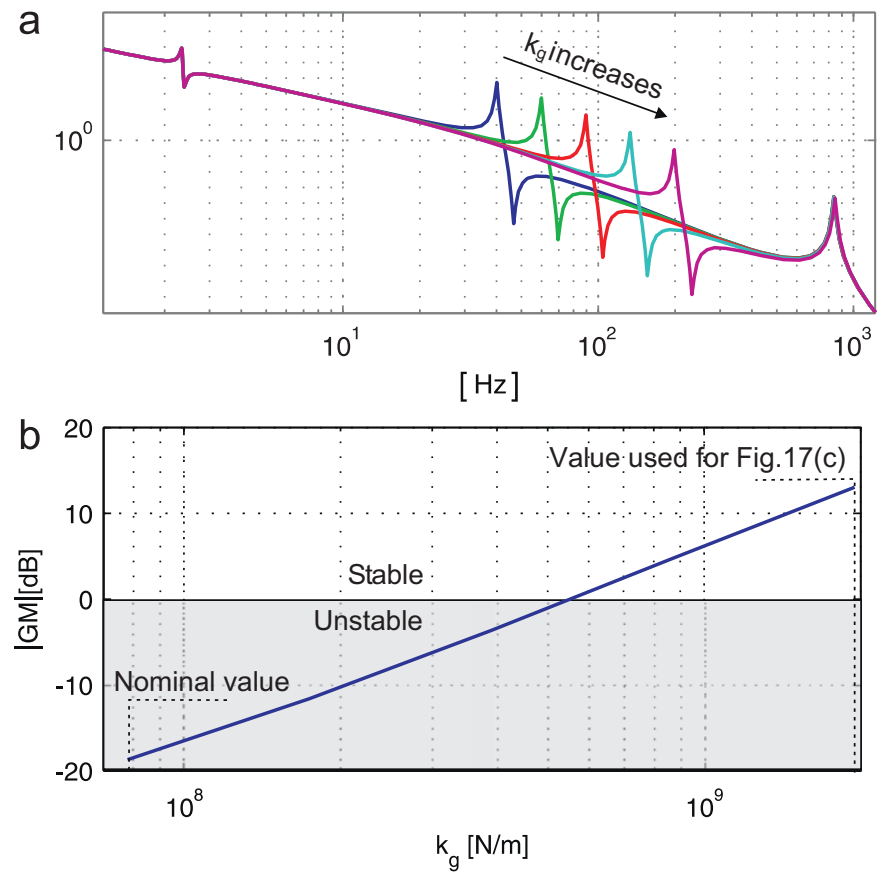

C

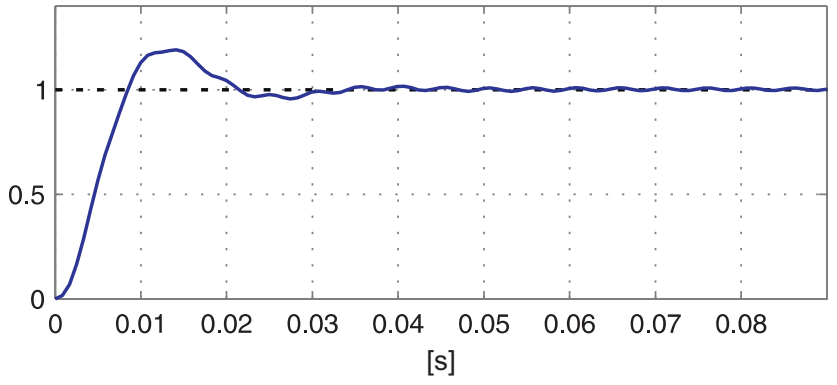

Fig. 17. Positioning the quadrupole: (a) open-loop transfer function between the actuator and the quadrupole displacement; (b) gain margin as a function of $k_{g}$; and (c) step response for the highest value of $k_{g}$.

an adaptive controller is presented, but the performances are always traded off by the Bode integral, between good rejection at low frequency and amplification in the mid frequency range around half of the repetition rate.

\subsection{Quadrupole positioning}

In this section, we briefly consider the possibility to use the final doublets as kickers, by mechanically moving them between each pulse (Figs. 1 and 2). In this case, QD0 have to be mounted on extremely stiff actuators, e.g. piezoelectric stack actuators. For example, let us consider that $k_{q}^{*}=5000 k_{q}=7.9 \mathrm{GN} / \mathrm{m}$. The force delivered by the actuator will be

$f^{+}=g h \Delta x$

where $g$ is the gain and $h$ is the control filter. Taking a simple integral controller, Fig. 17(a) shows the open-loop transfer function between the control force and the displacement of the quadrupole, where the nominal value of $k_{g}$ is gradually multiplied by a factor up to 5 . One clearly sees that to obtain a stable configuration and modify the position of the quadrupoles between each pulse (see Fig. 17(c)), an extremely stiff quadrupole under-support is required. If such an option can be used on a rigid ground, where the vibration level is low, it is not a realistic option for the final focus.

\section{Feed forward with vibration sensors}

In order to further improve the performances of the beam based feedback, one can in principle use the relative vertical displacement of the quadrupoles measured by the vibration sensors, and deflect the beams to minimize the beam-beam offsets. In this case, Eq. (27) becomes

$\Delta b(n)=H(n)\left[\Delta y(n)+n_{y}(n)\right]+F(n)\left[S(n) \Delta x(n)+n_{x}(n)\right]$

where $F(n)$ is the feed forward controller, $S(n)$ is the sensitivity of the vibration sensor and $n_{x}(n)$ its noise. Using Eq. (31) in Eq. (26), Eq. becomes (28)

$\Delta y(z)=\frac{1-F(z) S(z)}{1+H(z)} \Delta x(z)-\frac{H(z)}{1+H(z)} \Delta n_{y}(z)-\frac{F(z)}{1+H(z)} \Delta n_{x}(z)$.

The resulting $\Delta y$ is also shown in Fig. 16(a) and (b). It has been obtained with a proportional controller for $F(z)$, and the sensitivity curve of the seismometer Guralp $40 \mathrm{~T}$ [38]. The seismometer noise $n_{x}$, given by Eq. (15), is also shown for comparison.

This feed forward strategy is attractive, as it can in principle reduce the beam jitter down to the noise of the displacement sensor in the frequency range where the beam based feedback cannot work. However, the practical implementation is not straightforward, essentially because of system knowledge issues, and second order effects [39].

\section{Conclusions and future work}

In this paper, we have presented a simple model of the final focus of future linear collider. A key feature of the model is that it includes both the model of the beam and the structure, and that it has been updated using data measured in a representative accelerator environment. Using this model, several strategies to improve the stability have been objectively tested, and have led to important conclusions and guidelines for future designs. In summary, it has been shown that

- A pre-isolation of the whole final focus system can reduce beam jitter only if the mechanical vibrations of the final quadrupoles are caused by the ground motion (i.e. if the coherence between the two signals is very good), which is not the case for the rotating shielding of the CMS experiment, probably because of the high technical noise.

- For the same reason, an improvement of the structural damping has only a marginal effect on the reduction in the final quadrupoles vibrations.

- On the other hand, if a cantilever type structure is adopted, it has been found that a network of cables can drastically improve the robustness to the technical noise, and improve the stability of the final focus. Another interesting property of the active cables is that they can be used to realign the final quadrupoles, and replace the cam system currently foreseen.

- The controller implemented to actively improve the stability of the final quadrupoles consists of two independent systems (one for each side) chosen for simplicity and performances considerations.

- The dynamics of the cantilever does not provide a sufficient rigidity to use the quadrupoles as dipole correctors. As a consequence, it is better to adopt a soft strategy to actively stabilize the quadrupoles, and benefit from the passive isolation at high frequency.

- A second order controller has also been implemented to reduce the beam-beam offset. 
- A feed forward strategy has also been tested successfully. However, a more advanced treatment would be required to address the issues of system knowledge and non-linearities.

In a future work, the theoretical results presented in this paper will be transposed to design a full scale final focus system, including a finite element model of the structure, an active stabilization system, a network of cables, and a MIMO controller. An experimental validation is also planned on a quarter scaled test set-up, representing one half of the final focus.

\section{Acknowledgments}

The research leading to these results has been jointly funded by the European Commission under the FP7 Research Infrastructures project EuCARD, grant agreement No. 227579, and by the Brain Back to Brussels program from Brussels Capital Region for the first author. The authors also gratefully acknowledge Daniel Schulte for useful discussions on the beam dynamics, and the reviewers for their careful proofreading of the original manuscript and many useful comments.

\section{References}

[1] ILC Reference Design Report. Technical Report ILC-Report-2007-01, 2007.

[2] 〈http://clic-stability.web.cern.ch/clic-stability/〉.

[3] J.P. Delahaye, Towards CLIC feasibility, in: IEEE International Particle Accelerator Conference IPAC10, 23-25 May 2010, Kyoto, Japan, 2010.

[4] T. Suehara, M. Oroku, T. Yamanaka, H. Yoda, T. Nakamura, Y. Kamiya, Y. Honda, T. Kume, T. Tauchi, T. Sanuki, S. Komamiya, Nuclear Instruments and Methods in Physics Research Section A: Accelerators, Spectrometers, Detectors and Associated Equipment 616 (1) (2010) 1.

[5] R.W. Assmann, J.B. Jeanneret, A. Verdier, L. Vos, E. Wildner, F. Zimmermann, R. Brinkmann, C. Montag, I. Reyzl, N. Walker, C.E. Adolphsen, J. Frisch, N. Phinney, T.O. Raubenheimer, A. Seryi, P.G. Tenenbaum, Stability Considerations for Final Focus Systems of Future Linear Colliders, June 2000 oai:cds.cern.ch:455297.

[6] S. Redaelli, Stabilization of Nanometre-Size Particle Beams in the Final Focus System of the Compact LInear Collider (CLIC), Ph.D. Thesis, EPFL, Lausanne, 2003.

[7] J. Irwin, F. Zimmermann, Impact of Final-Focus Ground Motion on NLC Luminosity, June 1996, 4p., oai:cds.cern.ch:314906 (SLAC-PUB-7142).

[8] A. Seryi, L. Hendrickson, G. White, Issues of Stability and Ground Motion in ILC, Technical Report SLAC-PUB-11661, SLAC, Stanford, CA, February 2006.

[9] B. Parker, A. Mikhailichenko, K. Buesser, J. Hauptman, T. Tauchi, P. Burrows, T. Markiewicz, M. Oriunno, A. Seryi, Functional Requirements on the Design of the Detectors and the Interaction Region of an $\mathrm{e}+\mathrm{e}-$ Linear Collider with a Push-Pull Arrangement of Detectors. oai:cds.cern.ch:1239675, Technical Report EuCARD-CON-2009-026, 2009.

[10] I. Reyzl, Stabilization of Beam Interaction in the Tesla Linear Collider (DESYM-2000-04-ZM), November 2000, 3p.

[11] A. Sery, A. Mosnier, Physical Review E 56 (1997) 3558.

[12] T.O. Raubenheimer, F. Zimmermann, Reviews of Modern Physics 72 (January) (2000) 95.

[13] M. Aleksa, R.W. Assmann, W. Coosemans, G. Guignard, N. Leros, M. Mayoud, S. Redaelli, F. Ruggiero, S. Russenschuck, D. Schulte, I.H. Wilson, F. Zimmermann, The CLIC Study of Magnet Stability and Time-Dependent Luminosity Performance, July 2001, oai:cds.cern.ch:512353.
[14] A. Seryi, M. Breidenbach, J. Frisch, Ground Motion Studies and Modeling for the Interaction Region of a Linear Collider (physics/0008191. SLAC-PUB8594), 2000.

[15] L. Hendrickson, J. Frisch, T. Himel, A. Raubenheimer, A. Seryi, M. Woodley, G. White, Simulations of IP feedback and stabilization in the NLC, in: Proceedings of the EPAC 04, Lucerne, Switzerland, 2004.

[16] A. Seryi, Nuclear Instruments and Methods in Physics Research A 623 (2010) 23.

[17] H. Aihara, et al., SiD Letter of Intent, Technical Report arXiv:0911.0006 [physics.ins-det], 2009.

[18] T. Abe, et al., The International Large Detector: Letter of Intent, Technical Report arXiv:1006.3396 [hep-ex], 2010.

[19] F. Simon, Detector Systems at CLIC, ArXiv e-prints, September 2011.

[20] E. Courant, H. Snyder, Annals of Physics 3 (1957) 1.

[21] G. Stupakov, Quadrupole Misalignments and Steering in Long Linacs, Technical Report, SLAC-PUB-8694, 1985.

[22] A. Sery, O. Napoly, Physical Review E 53 (5) (1996) 5323.

[23] C. Collette, K.A. Artoos, M. Guinchard, A. Kuzmin, M. Sylte, F. Lackner, C. Hauviller, CLIC main beam quadrupoles stabilization, in: CLIC Workshop, Geneva, Switzerland, 2009.

[24] A. Kuzmin, Ground Vibration Measurements and Experiment Part Motion at CMS, Technical Report EDMS No. 1027459, 2009.

[25] A. Preumont, Random Vibration and Spectral Analysis, Kluwer Academic Publishers, 1994.

[26] A. Gaddi, H. Gerwig, N. Siegrist, F. Ramos, Dynamic Analysis of the ff Magnets Pre-isolator and Support System, Technical Report EDMS No. 1098581, 2010.

[27] C. Collette, S. Janssens, K. Artoos, C. Hauviller, Active vibration isolation of high precision machine (keynote lecture), in: Sixth International Conference on Mechanical Engineering Design of Synchrotron Radiation Equipment and Instrumentation (MEDSI 2010), Oxford, UK, 2010.

[28] L. Zuo, Element and System Design for Active and Passive Vibration Isolation, Ph.D. Thesis, Massachusetts Institute of Technology, November 2004.

[29] J. Frisch, A. Chang, V. Decker, E. Doyle, L. Eriksson, L. Hendrickson, T. Himel, T. Markiewicz, R. Partidge, A. Seryi, Vibration stabilization of a mechanical model of a $\mathrm{x}$-band linear collider final focus magnet, in: 22nd International Linear Collider Conference, Germany, 16-18 August 2004.

[30] B. Bolzon, Etude des vibrations et de la stabilisation à l'échelle sousnanométreique des doublets finaux d'un collisionneur linéaire, Ph.D. Thesis, University of Savoie, 2007.

[31] A. Preumont, Vibration Control of Active Structures: An Introduction, third ed., Kluwer Academic Publishers, Dordrecht (The Netherlands), 2011.

[32] F. Lackner, K. Artoos, C. Collette, H. Mainaud Durand, C. Hauviller, J. Kemppinen, R. Leuxe, Development of an excentric cam based active prealignment system for the CLIC main beam quadrupole magnet, in: Proceedings of the Sixth International Workshop on Mechanical Engineering Design of Synchrotron Radiation Equipment and Instrumentation (MEDSIO0), Oxford, United Kingdom, 2010.

[33] S. Allison, L. Eriksson, L. Hendrickson, T. Himel, A. Seryi, Active vibration suppression $r+d$ for the next linear collider, in: Proceedings of the Particle Accelerator Conference, Chicago, 2001.

[34] J. Frisch, L. Hendrickson, T. Himel, A. Seryi, Active vibration suppression $r \& d$ for the nlc, in: Proceedings of Eighth International Conference on Accelerator and Large Experimental Physics Control Systems (ICALEPCS 2001), San Jose, CA, 2001.

[35] R. Greenall, Nanovibration Control, University of British Columbia, 2001.

[36] C. Collette, S. Janssens, K. Artoos, A. Kuzmin, P. Fernandez-Carmona, M. Guinchard, R. Leuxe, C. Hauviller, Nuclear Instruments and Methods in Physics Research Section A: Accelerators, Spectrometers, Detectors and Associated Equipment 643 (1) (2011) 95

[37] B. Caron, G. Balik, L. Brunetti, A. Jeremie, Vibration Control of the Beam of the Future Linear Collider, 2011, in2p3-00638748.

[38] Guralp, Guralp catalogue.

[39] Y. Renier, Possibility to detect ground motion at ATF2, in: International Workshop on Future Linear Colliders, Granada, Spain, 2011. 